\title{
Leptin and hyperleptinemia - from friend to foe for cardiovascular function
}

\author{
J Ren \\ Division of Pharmaceutical Sciences and Graduate Neuroscience Program, University of Wyoming College of Health Sciences, Laramie, Wyoming \\ 82071-3375, USA \\ (Requests for offprints should be addressed to J Ren; Email: jren@uwyo.edu)
}

\begin{abstract}
The obese gene product, leptin, plays a central role in food intake and energy metabolism. The physiological roles of leptin in human bodily function have been broadened over the past decade since leptin was first discovered in 1994. Evidence has suggested that leptin plays a specific role in the intricate cascade of cardiovascular events, in addition to its well-established metabolic effects. Leptin, a hormone linking adiposity and central nervous circuits to reduce appetite and enhance energy expenditure, has been shown to increase overall sympathetic nerve activity, facilitate glucose utilization and improve insulin sensitivity. In addition, leptin is capable of regulating cardiac and vascular contractility through a local nitric oxidedependent mechanism. However, elevated plasma leptin levels or hyperleptinemia, have been demonstrated to correlate with hyperphagia, insulin resistance and other
\end{abstract}

markers of the metabolic syndrome including obesity, hyperlipidemia and hypertension, independent of total adiposity. Elevated plasma leptin levels may be an independent risk factor for the development of cardiovascular disease. Although mechanisms leading to hyperleptinemia have not been well described, factors such as increased food intake and insulin resistance have been shown to rapidly enhance plasma leptin levels and subsequently tissue leptin resistance. These findings have prompted the speculation that leptin in the physiological range may serve as a physiological regulator of cardiovascular function whereas elevated plasma leptin levels may act as a pathophysiological trigger and/or marker for cardiovascular diseases due to tissue leptin resistance.

Journal of Endocrinology (2004) 181, 1-10

\section{Introduction}

Obesity is an increasingly prevalent metabolic disorder affecting not only the developed but also the developing countries. The pathogenesis of obesity is multifactorial incorporating both genetics and lifestyle. While heredity explains $30 \%$ to $70 \%$ of obesity cases with causative genetic mutations being identified in several recognizable pleiotropic obesity syndromes such as the Bardet-Biedl syndrome and melanocortin 4 receptor (MC4R) gene mutation-associated obesity (Farooqi et al. 2003), the contribution from lifestyle factors such as diet and satiety may be predominantly responsible for the recent dramatic increase in the prevalence of obesity. In the United States, despite the fact that consumption of fat has been reduced dramatically over the last three decades, a decrease in incidence of obesity has not occurred. This is likely attributable to maintenance of food intake with an increase in total calories and also to reduced physical activity.
Uncorrected obesity, when sustained, often leads to cardiac hypertropy, ventricular dysfunction, reduced diastolic compliance, increased blood pressure, arteriosclerosis and other vascular complications. In addition, uncorrected obesity dramatically enhances the propensity of a cluster of metabolic diseases such as diabetes mellitus, insulin resistance, hyperinsulinemia, hyperlipidemia, hyperuricemia and low plasma high-density lipoprotein cholesterol, collectively known as the metabolic syndrome (Sowers 1998, Unger \& Orci 2002). Although most of the comorbidities relating obesity to cardiovascular dysfunction become more intense as body mass index (BMI) increases in conjunction with body fat distribution, longterm longitudinal studies indicate that obesity not only relates to, but also independently predicts, certain cardiovascular defects such as coronary atherosclerosis (Sowers 1998). This relationship appears to exist for both men and women with minimal increases in BMI (Sowers 1998). Nevertheless, no clear culprits have been identified to link 
any product of mutant gene or lifestyle factors to disrupted cardiovascular function under obesity. Although it is generally accepted that activation of the sympathetic autonomic nervous system and impairment of endothelial function are the two pivotal phenotypical traits in obesity (Steinberg et al. 1996, Julius et al. 2000, Kuo et al. 2003), the cellular mechanisms responsible for sympathetic hyperactivity and endothelial dysfunction have not yet been clearly elucidated. To add to the complexity, metabolic syndromes such as diabetes and hypertension often coexist with obesity and may themselves exert independent detrimental effects on cardiovascular function. Therefore, the direct and independent impact as well as the consequence of obesity on cardiovascular function may be largely obscured.

Obesity has long been considered as a behavioral and social disorder. It was not until 1994 that the discovery of the $16 \mathrm{kDa}$ obese gene $(\mathrm{ob})$ product, leptin, brought a complete revolution to the concept of obesity (Zhang et al. 1994, Caro et al. 1996, Leibel et al. 1997, Friedman \& Halaas 1998). Released by adipose tissue, leptin is considered the hormonal signal bridging the peripheral adipose tissue to the central nervous system (CNS) for the control of appetite and energy expenditure (Campfield et al. 1995, Caro et al. 1996, Friedman 2002). The CNS action of leptin is crucial to maintain energy balance likely through an increased sympathetic tone (Pelleymounter et al. 1995, Havel 2000, Hall et al. 2002). Moreover, localization of leptin receptors in a wide range of tissues has indicated central as well as peripheral actions of leptin (Bjorbaek et al. 1997, Hall et al. 2002, Jequier 2002). For example, intravenous leptin infusion has been shown to enhance norepinephrine turnover and sympathetic nerve activity (Haynes et al. 1997). Interaction with both CNS and peripheral nerves under different conditions reflects the complexity of leptin signaling in the regulation of bodily function. Leptin may act as an endocrine, a paracrine, as well as an autocrine factor. It participates in multiple regulatory mechanisms such as energy expenditure/metabolism, cell proliferation/differentiation, as well as signal interaction with other hormonal regulators of energy and metabolism such as insulin, insulin-like growth factors, growth hormone, glucagon and glucocorticoids (Friedman 2002, Hall et al. 2002, Margetic et al. 2002, Sweeney 2002). The leptin signaling system is becoming ever more complex than was first envisioned. This review will be focused on the literature dealing with the cardiovascular response of leptin, predominantly on those articles which have appeared in the last five years. It is crucial to enrich our understanding of the complex interactions among leptin, hyperleptinemia and cardiovascular function to understand the pathogenesis of the obesity-related cardiovascular dysfunction so that optimal management and therapy may be achieved for obese individuals who are at high risk of cardiovascular events.

\section{Leptin, leptin receptors and their biological actions}

Leptin has structural homology to tumor necrosis factor alpha (TNF- $\alpha$ ), interleukin 6 (IL-6), leukemia inhibitory factor, granulocyte-colony stimulating factor, glycoprotein 130 (gp130) and other cytokine family proteins, and is therefore considered a cytokine-like substance (Ahima \& Flier 2000, Sweeney 2002). Nuclear magnetic resonance and crystal structure analysis revealed a four-helix bundled structure for leptin with four antiparallel $\alpha$-helices linked with two long crossover arms and one short loop. Binding of leptin to its membrane receptor results in homodimerization. Five isoforms of leptin receptors have been identified so far, namely Ob-Ra, Ob-Rb, Ob-Rc, $\mathrm{Ob}-\mathrm{Rd}$ and $\mathrm{Ob}-\mathrm{Re}$. The leptin receptor is a transmembrane protein with structural homology to that of the gp130 receptor family. The extracellular domain of the leptin receptor contains two cytokine binding regions with one region being the specific leptin-binding site. The transmembrane domain consists of 23 amino acids and the intracellular domain is variable in length. The intracellular domain is usually associated with one or two box motifs, whereas most gp130 receptors have three box motifs. The cytoplasmic motifs allow leptin receptors to interact with intracellular messengers such as mitogen-activated protein (MAP) kinase, insulin receptor substrate (IRS-1 and IRS-2), nitric oxide (NO), Janus kinase (JAK) and signal transducer and activator transcription factor (STAT) (Ahima \& Flier 2000, Sweeney 2002). While the box 1 motif is necessary for the short form of leptin receptor (Ob-Rs) activation of JAK2, the box 2 motif is required for full transduction of the JAK-STAT signal (Bjorbaek et al. 1997). Upon binding of leptin, the Ob-Rb receptor has been demonstrated to activate STAT1, STAT3, STAT5, and STAT6 in vitro but only STAT3 in vivo. The downstream signaling of STAT is not completely understood at this time but has been somewhat revealed in the heart, brain and other cell lines (Bjorbaek et al. 1997). Earlier studies identified the MAP kinase pathway as a downstream signaling mechanism of Ob-Rs (Bjorbaek et al. 1997). In addition, the extracellular signal regulated kinase (ERK) was activated with leptin stimulation. While this is enlightening for $\mathrm{Ob}-\mathrm{Rs}$, the long form $\mathrm{Ob}-\mathrm{Rb}$ receptor has also been demonstrated to stimulate ERK activity although at a much higher potency (Bjorbaek et al. 1997). In vascular endothelial cells, leptin can activate the stress activated protein kinase (SAPK) and Jun NH2terminal kinase (JNK) transduction pathways of the MAP kinase family (Bouloumie et al. 1999). Leptin may also up-regulate the transcription factor activation protein 1 (AP-1) (Bouloumie et al. 1999). While the SAPK/JNK pathway appears to be associated with leptin signaling, it may be directly activated by reactive oxygen species (ROS), thus making sustained hyperleptinemia potentially hazardous. Activation of SAPK/JNK may contribute to the pro-oxidant effects of leptin, especially at chronic high 
circulating levels (Bouloumie et al. 1999, Anderson \& Ren 2002).

Accumulating evidence has suggested a close interaction between leptin signaling and insulin signaling, or between hyperleptinemia and hyperinsulinemia (Ookuma et al. 1998). Obese individuals may often display both hyperleptinemia and hyperinsulinemia concurrently, although neither is likely to be permissive to the onset of the other comorbidity (Naderali et al. 2001, Hintz et al. 2003). Binding of leptin to its receptors directly enhances the activity of IRS-1 and IRS-2 (Bjorbaek et al. 1997, Kellerer et al. 1997). Phosphorylation of IRS on the serine/threonine residue may lead to decreased phosphatidylinositol-3 (PI-3) kinase and subsequently deficient PI-3 kinase activation (Bjorbaek et al. 1997, Kolter et al. 1997, Zhao et al. 1998, Harvey et al. 2000). Therefore, hyperleptinemia may be associated with suppressed PI-3 kinase activity and reduced cardiovascular inotropicity since PI-3 kinase is a known positive inotropic mediator for the heart and vasculature (Northcott et al. 2002, von Lewinski et al. 2003). This notion is supported by our recent evidence indicating that the leptin-induced cardiac depressant effect was abolished by the JAK2 inhibitor AG-490 (Wold et al. 2002). It may be speculated that leptin phosphorylates IRS-1, IRS-2 or both, by first activating JAK2, an upstream signaling molecule of PI-3 kinase activation (Sweeney 2002), which may eventually lead to drained PI-3 activity.

Leptin plays a pivotal role in a wide variety of organ systems including the reproductive, renal and cardiovascular systems (Ahima \& Flier 2000, Friedman 2002). Leptin influences the central regulation of food intake and energy expenditure via cerebral leptin receptors. Overeating (hyperphagia) may lead to an elevation whereas fasting or caloric restriction results in a fall in leptin levels (Wang et al. 2001). A fall in leptin levels (such as in starvation and malnutrition) may lead to immune deficiency and impaired host defence including lymphoid atropy and T-lymphocyte dysfunction, which can be restored with leptin supplementation. Reduction in plasma leptin may trigger CNS adaptive responses to starvation by suppressing reproductive and thyroid function (Mancuso et al. 2002). Metabolically, leptin promotes the redistribution of intrahepatic glucose fluxes with an increase in gluconeogenesis and a parallel decrease in glycogenolysis (Kamohara et al. 1997, Friedman 2002). The signaling mechanism in glucose and energy metabolism is quite different between leptin and insulin although both hormones act on receptors in the same regions of the hypothalamus. Although leptin and insulin may both reduce appetite, the two hormones may combine subadditively in the acute regulation of food intake (Air et al. 2002, Kuo et al. 2003). While the satiety factor, leptin, facilitates energy expenditure, insulin stimulates glucose uptake for energy utilization or storage. The interaction between leptin and insulin is quite complex.
Release of leptin may be significantly increased when adipocytes are exposed to insulin and glucose (Mueller et al. 1998, Levy \& Stevens 2001), which underscores the phenomenon of hyperleptinemia in type 2 diabetes. On the other hand, an increase in plasma leptin levels has been shown to reduce insulin release and enhance insulin sensitivity (Barzilai et al. 1997, Ookuma et al. 1998, Cases et al. 2001). Although short-term dietary obesity has been shown to induce endothelial dysfunction without causing insulin resistance (Naderali et al. 2001), long-term hyperphagia or hyperleptinemia may eventually down-regulate insulin signaling and induce insulin resistance (Anderson \& Ren 2002). It may be speculated that sustained hyperleptinemia-induced over-phosphorylation of IRS-1/ IRS-2 and subsequently deficient PI-3 kinase activity may play a role in the onset of insulin resistance (Zhao et al. 1998, Harvey et al. 2000). The role of leptin in insulin release and action is still debatable with conflicting observations. It is believed that the dose and duration of leptin exposure may be a critical factor in determining the action or sensitivity of insulin.

\section{Regulation of leptin levels by insulin and obesity}

Plasma leptin levels are strongly correlated with adiposity. Tissue adiposity, along with gender, is the main determinant of leptin gene expression and release (Trayhurn et al. 1998). Change in plasma leptin levels and/or leptin signaling has a profound pathological impact on body weight control. Both leptin deficiency and leptin receptor defect are sufficient to produce obesity of genetic origin (Unger \& Orci 2002). However, the most common trigger of obesity is not genetic defects of leptin or its receptor but rather depends on overeating or high fat diet intake, which may rapidly increase plasma leptin levels (Friedman \& Halaas 1998, Wang et al. 2001). Although it has been speculated that hyperleptinemia leads to tissue resistance of leptin and insulin (Frederich et al. 1995), how hyperleptinemia and/or impaired leptin signaling triggers cardiovascular dysfunction is unclear and will be further discussed in the following sections. Several factors have been identified as participating in the regulation of leptin synthesis and release including the sympathetic nervous system, insulin and body adiposity (Havel 2000). Sympathetic nervous system activity is believed to be a key inhibitor of leptin release. Catecholamines including norepinephrine, epinephrine and isoprenaline have all been shown directly to inhibit leptin synthesis (Trayhurn et al. 1998, Evans et al. 1999). Paradoxically, leptin may directly activate sympathetic outflow within the hypothalamus and stimulate adrenal medullary release of epinephrine, thus creating a negative feedback loop between leptin and the sympathetic nervous system (Eikelis et al. 2003).

Plasma leptin levels appear to be associated with the propensity of certain cardiac damage in patients with acute 
myocardial infarction (Fujimaki et al. 2001). Elevated plasma leptin levels and its soluble receptor were found in patients with congestive heart failure (Christian Schulze et al. 2003, Schulze et al. 2003), suggesting that leptin may participate in the catabolic cardiac cachexia en route to congestive heart failure. On the other hand, plasma leptin levels were reduced in patients with idiopathic sympathetic nerve degeneration and low sympathetic tone. However, intravenous infusion of the central sympathetic nervous system inhibitor, clonidine, reduced sympathetic nervous activity without affecting plasma leptin levels. This apparently discrepant response may be related to decreased renal clearance of leptin as a result of clonidine-induced reduction in renal blood flow.

There seems to be a positive correlation among proinsulin, insulin and leptin levels, all of which were elevated in hypertensive patients when indexed for body size (Malmqvist et al. 2002). A significant negative correlation was found between the levels of leptin and IL-6. In a multivariate analysis, plasma leptin levels were found in correlation with diet, independent of age, BMI, body fat, alcohol consumption or insulin. Fish eaters exhibited lower plasma leptin levels than vegetable eaters with similar BMI (Winnicki et al. 2002). This is consistent with the epidemiological indication of reduced cardiovascular risk with a high-fish diet.

\section{Leptin, vascular function and blood pressure}

Like other metabolic hormones such as insulin, leptin possesses potent vascular effects and participates in the regulation of sympathetic tone and arterial blood pressure (Haynes et al. 1997). The regulatory effects on vascular tone and blood pressure have been demonstrated by both intracerebroventricular and intravenous administration of leptin, indicating central as well as peripheral actions of the hormone. Intracerebroventricular administration of leptin was found to elicit an increase in arterial blood pressure (Matsumura et al. 2000), consistent with its sympathoexcitatory property. The pressor effect of leptin was proportional to its levels in the cerebrospinal fluid (Shirasaka et al. 2003), suggesting that the pressor and sympathoexcitatory properties of leptin are attributed to a central mechanism. This is supported by the fact that leptin administered intravenously or intracerebroventricularly significantly increases the sympathetic outflow to kidneys, adipose tissue, skeletal vasculature and adrenal medulla in rodents. Interestingly, the dose of leptin required to enhance catecholamine release is at least 100 times higher for intravenous than for intracerebroventricular injection (Satoh et al. 1999), suggesting that leptin activates sympathetic outflow mainly via a central mechanism. On the other hand, chronic intravenous infusion of leptin also significantly enhanced arterial blood pressure associated with an elevated circulating plasma leptin level
(Shirasaka et al. 2003). The intravenous infusion of leptin induced an increase in blood pressure in conjunction with overt weight loss, which itself is expected to lower arterial blood pressure (Shek et al. 1998). The hypertensive effect of chronic intravenous leptin infusion disappeared when leptin infusion was stopped. It should be mentioned that acute leptin administration does not consistently elicit a hypertensive effect (Haynes et al. 1997, Shirasaka et al. 2003). Such discrepancy may be attributed to the small and inconsistent changes in plasma leptin following bolus injection or short-term infusion of leptin. Although accumulating evidence suggests a naturalistic linkage between sympathetic activity and plasma leptin levels, certain mechanisms other than leptin, particularly hyperinsulinemia, have been suggested to be responsible for the enhanced sympathetic activation in obesity. Further study is warranted to examine the interaction between leptin and other signaling pathways in the elevated sympathetic nervous activity in obesity.

In addition to its central neuronal sympathoexcitatory action, leptin may exert direct peripheral vascular actions on certain vasoactive mediators such as $\mathrm{NO}$ and endothelin-1 (ET-1). Leptin has been shown directly to induce vasorelaxation through $\mathrm{NO}$-dependent as well as NO-independent mechanisms. Leptin elicits an NOdependent arterial vascular relaxation with the chloride ion being an important regulator in leptin-induced endothelial NO release (Kimura et al. 2000). Leptin has been shown to stimulate endothelial NO synthesis, upregulate ET-1 production and promote accumulation of ROS in human umbilical vein endothelial cells (Bouloumie et al. 1999, Lembo et al. 2000, Yamagishi et al. 2001, Quehenberger et al. 2002, Vecchione et al. 2002). The leptin-induced vascular NO production was further proven to be mediated through PI-3 kinase-independent endothelial nitric oxide synthase (eNOS) phosphorylation by the Akt pathway (Vecchione et al. 2002). Recent evidence also indicated the possible involvement of AMP-activated protein kinase (AMPK) in enhanced eNOS phosphorylation and NO production in response to leptin. AMPK, activated by an increase in the AMP-to-ATP ratio, and/or a decrease in phosphocreatine, is known to mediate the metabolic effects of leptin including stimulation of glucose uptake and fatty acid oxidation (Minokoshi et al. 2002). AMPK has been shown to promote phosphorylation of eNOS either independently or in concert with Akt in a number of cell types including endothelial cells and myocytes (Chen et al. 1999), thus providing an important regulatory link between metabolic stress and cardiovascular function. What makes AMPK more attractive is the recent observation of elevated expression and phosphorylation of AMPK following chronic leptin treatment (Steinberg et al. 2003). It is therefore reasonable to postulate that leptin-induced regulation of $\mathrm{NO}$ in the cardiovascular system may be related to the enhanced expression and activation of AMPK. Nevertheless, the 
precise role of $\mathrm{NO}$ in cardiovascular regulation elicited by leptin still remains ambiguous. Leptin was shown to induce an acute hypotensive effect in 6-hydroxydopamine sympathectomized rats, which was unaffected by NOS inhibition, suggesting a predominant role of the endothelium-derived hyperpolarizing factor mechanism in the hypotensive effect of leptin (Lembo et al. 2000). The direct vascular response of leptin in rodents was further confirmed in humans. Leptin directly induces vasodilation through an NO-independent pathway in healthy men (Nakagawa et al. 2002). The discrepancy in the NO dependence on the vascular effect of leptin is still largely unclear but may be related to the source of leptin (human versus murine), species, and the nature of the vascular beds tested. Considering pressor (sympathetic activation) and depressor (increased NO) actions, the integrated mechanism of actions of leptin on vascular tone and arterial blood pressure regulation is rather complex. State-of-the-art transgenic models of leptin and its receptor have provided some useful information regarding the vascular regulatory role of leptin. Transgenic mice overexpressing leptin displayed high arterial blood pressure compared with wild-type control mice, despite decreases in body fat (Aizawa-Abe et al. 2000). The increase in blood pressure was accompanied by an increase in urinary norepinephrine excretion and was normalized after $\alpha$-adrenergic or ganglionic blockade (Aizawa-Abe et al. 2000). These observations support the sympathetic pressor property of leptin (Shek et al. 1998, Haynes 2000). However, it was recently reported that moderate hyperleptinemia produced by adenoviral gene transfer to obese mice reduced body weight without affecting blood pressure (Zhang et al. 2002). On the other hand, the leptin-deficient ob/ob obese mice showed slightly lower arterial blood pressure than their wild-type controls, despite profound obesity that would be expected to increase blood pressure. Administration of leptin to the ob/ob mice (the 'leptin reconstitution') resulted in elevated blood pressure despite reduced food intake and body weight (Aizawa-Abe et al. 2000, Mark \& Sivitz 2002). In contrast to the leptin-deficient $\mathrm{ob} / \mathrm{ob}$ mice, agouti yellow obese mice display partial leptin resistance, compensatory hyperleptinemia and elevated blood pressure (Mark et al. 1999, Correia et al. 2002). One other interesting note is that the vascular response of leptin seems to be independent of that of insulin or insulin resistance. Dietary obesity, which itself is associated with hyperleptinemia and leptin resistance (Wang et al. 2001), induces endothelial dysfunction without causing insulin resistance (Naderali et al. 2001). Studies from our laboratory, on the other hand, indicated that insulin resistance itself is not permissive to, or a prerequisite for the onset of cardiac leptin resistance (Hintz et al. 2003). It may thus be speculated that leptin and insulin possess distinct signaling mechanisms in the regulation of vascular tone.

Leptin receptors are localized in the hypothalamus and peripheral vascular tissues such as endothelial cells and platelets (Lembo et al. 2000). The hypothalamic receptors for leptin are expected to assist the transfer of the hormone across the blood-brain barrier. The transfer is believed to occur at a low rate. Therefore, the levels of leptin in the cerebrospinal fluid are much lower than those in the plasma. The central leptin action is usually associated with elevated cerebrospinal leptin and an increased JAK/STAT3 signaling, leading to increased sympathetic tone and reduced food intake. JAK/STAT and IRS-2 may mediate leptin-induced activation of PI-3 kinase (Kellerer et al. 1997), which is a critical signal regulating vascular tone (Northcott et al. 2002). The specific pathway may be activation of JAK-2 by leptin followed by tyrosine phosphorylation of IRS-2 which would result in an increase in PI-3 kinase activity. The enhanced sympathetic nervous tone is thought to play a role in the cardiovascular response to leptin. Although this may be the case, the contribution from the peripheral response to leptin vascular activity and arterial blood pressure cannot be ignored. While the enhanced sympathetic nervous activation is expected to increase the vascular tone and blood pressure, leptin has been shown to elicit peripheral vascular relaxation mediated by NO-dependent or -independent mechanisms when the sympathetic control is removed (Bjorbaek et al. 1997, Fruhbeck 1999, Kimura et al. 2000, Lembo et al. 2000). The leptin-induced peripheral NO release and vasorelaxation may serve to counter-balance the enhanced sympathetic activity in response to leptin. While there was an increase in lumbar sympathetic nervous activity in response to low physiological levels of leptin, no acute response in blood pressure or heart rate was observed (Mitchell et al. 2001).

\section{Leptin, heart rate and cardiac function}

While acute leptin infusion failed significantly to alter the heart rate over a span of 90 min (Fruhbeck 1999), chronic leptin treatment over one week and cerebral injection of leptin elicited a significant increase in heart rate along with an increase in sympathetic nervous activity (Shek et al. 1998). An astonishing positive correlation between hyperleptinemia and tachycardia has been confirmed in mildly obese or mildly hypertensive human subjects (Narkiewicz et al. 1999). While an increase in heart rate may enhance cardiac output and provide short-term beneficial effects, sustained tachycardia may cause cardiac hypertropy and ultimately heart failure. The higher heart rate in the hyperleptinemic individuals will impose a greater myocardial workload and therefore predispose the heart to pathophysiological changes, leading to congestive heart failure and myocardial infarction. This is supported by the clinical and experimental observations of obesity-induced cardiac hypertropy, and the direct correlation between BMI and the left ventricular size in human and animal models (Sowers 1998, Ren et al. 2000). Such a relationship 
may be transposed into a direct correlation between left ventricular size and plasma leptin levels. While it seems reasonable that the higher heart rate under hyperleptinemia may be due to leptin-induced sympathetic activation, an independent association between leptin levels and heart rate was observed in heart transplant recipients with sympathetic denervation (Winnicki et al. 2001). This finding suggests a direct effect of leptin on heart rate conceivably through cardiac leptin receptors, although direct effects of leptin on the cardiac conducting system and cardiac growth still warrant further study.

Leptin has been demonstrated to elicit concentrationdependent negative inotropic effects on myocyte contraction and intracellular $\mathrm{Ca}^{2+}$ release (Nickola et al. 2000). These effects were proven to be NO-dependent. However, the effects were blunted in ventricular myocytes from SHR hypertensive rats. A similar protein expression of the leptin receptor (Ob-R) was demonstrated between the WKY normotensive and the SHR hypertensive animals. Interestingly, leptin elicited overt post-receptor signaling pathway STAT3 phosphorylation in WKY but not SHR myocytes which was probably related to an up-regulated basal phosphorylation of STAT3 in SHR myocytes (Wold et al. 2002). The leptin-induced negative cardiac contractile response was blunted by the JAK2 inhibitor, AG-490, or the MAP kinase inhibitor, SB203580 (Wold et al. 2002), suggesting involvement of JAK2/STAT and p38 MAP kinase in the leptin-induced cardiac response. It is possible that NO may serve as the downstream mediator or the final instigator for JAK2/ STAT and p38 MAP kinase in cardiac myocytes. Our earlier evidence suggested that leptin increases the NOS activity in cardiac myocytes (Nickola et al. 2000). However, the leptin-induced increase in NOS activity was blunted under hypertension (Wold et al. 2002), which may be directly responsible for the loss of the leptin response in SHR myocytes.

Other signaling pathways have also been implicated in the leptin-induced cardiac effect. The adenylate cyclase complex has been shown to be affected by leptin in the H9c2 cardiac cell line. Differences in adenylate cyclase activity after short- and long-term exposure to leptin and the interaction between leptin and the sympathetic nervous system catecholamine neurotransmitters is believed to play a key role in the development of hypertension and congestive heart failure in obese patients (Illiano et al. 2002). Leptin has also been demonstrated to activate fatty acid oxidation and decrease triglyceride content without affecting glucose oxidation over a $60-\mathrm{min}$ perfusion period. Although leptin did not affect cardiac work, it increased oxygen consumption and decreased cardiac efficiency (Atkinson et al. 2002). The role of leptin in lipid metabolism is of interest and it may participate in the cardiac regulation of the hormone. Lipoapoptosis is a metabolic cause of tissue injury and death under obesity. As mentioned earlier, leptin is capable of oxidizing excessive long-chain fatty acids to improve cardiac function. However, the leptin-induced fatty acid oxidation may become inefficient under hyperleptinemia or leptin resistance, allowing unoxidized fatty acids to enter nonoxidative pathways, eventually leading to cellular injury (Unger \& Orci 2002). The ceramide/sphingosine pathway has been implicated in the lipoapoptosis of $\beta$-cells and cardiac myocytes of genetically obese rats under which condition the leptin malfunctions. The leptin-induced liporegulation serves to prevent lipid accumulation and lipoapoptosis. Disruption of liporegulation in response to leptin is speculated to be essential under hyperleptinemia and may attribute to the metabolic syndrome in obesity (Unger \& Orci 2002).

Circulating leptin levels have been postulated to be a marker of the cardiovascular and neuroendocrinological stress response. Patients with advanced congestive heart failure exhibit elevated plasma levels of leptin and its soluble receptor, indicating that leptin may participate in the catabolic state leading to the development of cardiac cachexia (Schulze et al. 2003). Critically ill patients display elevated leptin levels in response to stress-related cytokines (TNF- $\alpha$, interleukins) and loss of the circadian rhythm in leptin secretion, suggesting that the hormone plays a role in cardiac stress response (Modan-Moses et al. 2001). Cardiopulmonary bypass surgery was found to be associated with acute changes in circulating leptin levels inversely related to those of cortisol (Modan-Moses et al. 2001). Patients undergoing major surgery show a similar increase in plasma leptin levels compared with those following cardiopulmonary bypass, indicating that a sepsislike inflammatory syndrome does not further increase elevated leptin concentrations following major surgery (Dotsch et al. 2001).

\section{Leptin and circulatory function}

High levels of leptin are believed to be associated with lower arterial distensibility, an index of circulatory function relevant to the atherosclerotic process through mechanisms other than vascular relaxation (Singhal et al. 2002). Leptin promotes angiogenesis, regulates osteoblastic differentiation, enhances the calcification of vascular cells and potentiates the pro-thrombotic platelet aggregation through a novel leptin receptor-dependent mechanism (Sierra-Honigmann et al. 1998, Konstantinides et al. 2001, Parhami et al. 2001). This is consistent with the observation that leptin-deficient $\mathrm{ob} / \mathrm{ob}$ mice display delayed thrombus formation after arterial injury, which may be canceled by leptin replacement (Konstantinides et al. 2001). Obese individuals possess higher plasma levels of pro-thrombotic factors such as fibrinogen, von Willebrand factor, factor VII and plasminogen activator inhibitor-1 (PAI-1), which impose a higher risk of thrombosis and arteriosclerosis. The levels of pro-thrombotic factors and 


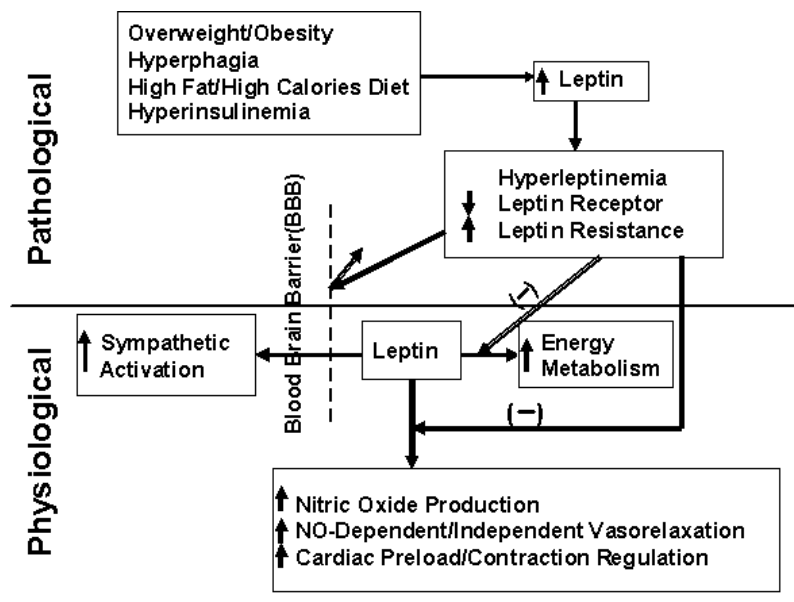

Figure 1 Regulation of the sympathetic, metabolic and cardiovascular functions of leptin under physiological 'euleptinemic' (lower half) or pathological 'hyperleptinemic' (upper half) conditions. Note that leptin cannot go through the blood-brain barrier (BBB) and therefore overweight, hyperphagia and hyperinsulinemia are unlikely to down-regulate leptin receptors and trigger leptin resistance in the CNS. However, the peripheral metabolic and cardiovascular actions of leptin will be interrupted by leptin resistance. $(-)$, inhibition or interruption.

PAI-1 are directly correlated with fat composition and therefore leptin levels. Paradoxically, higher plasma levels of anti-thrombotic factors such as tissue-type plasminogen activator and protein $\mathrm{C}$ have also been demonstrated in obesity to counteract the increased pro-thrombotic factors, probably through a compensatory mechanism. It is speculated that leptin-associated secretion of TNF- $\alpha$ and IL-6 from the adipose tissue may be responsible for the enhanced thrombosis formation (De Pergola \& Pannacciulli 2002), which predisposes to the pathogenesis of endothelial dysfunction and coronary heart disease. Similar to the common anti-obese measures, dietary (i.e. low-fat, high-fiber diet) and lifestyle (i.e. physical activity) factors seem to effectively lower these obesity-associated pro-thrombotic risks (De Pergola \& Pannacciulli 2002).

\section{Leptin/Leptin Resistance: an Anti-/Pro-Obesity Pair?}

A schematic diagram depicting the neuronal regulatory, cardiovascular and metabolic effects of leptin under both physiological and hyperleptinemic pathological conditions is summarized in Fig. 1. Using plasma norepinephrine spillover as a criterion, plasma leptin levels are a stronger predictive factor for obesity than percentage body fat, suggesting that hyperleptinemia is the prime drive underlying the sympathetic stimulation and pathophysiology of obesity-related cardiovascular dysfunctions (Grassi et al. 1998, Anderson \& Ren 2002). While leptin may be considered as an anti-obesity hormone due to its metabolic properties, hyperleptinemia and subsequently leptin resistance have surely made the ugly switch or transition. It is unlikely that leptin has evolved to counteract obesity since overweight individuals often become resistant to leptin action. On the other hand, due to the poor transport of leptin across the blood-brain barrier, hyperleptinemia cannot effectively signal the CNS to initiate sufficient negative feedback inhibition of the leptin signaling (Bjorbaek et al. 1999). While secretion of leptin may not be acutely determined by a single meal, a more chronic stimulus of food intake does modulate and affect leptin secretion. Elevated plasma leptin levels and severe resistance to leptin and insulin have been shown to develop after only 3 days of overfeeding. With the presence of insulin clamps, glucose production was decreased by $70 \%$ in control rats and by $28-53 \%$ in overfed rats. Similarly, leptin infusion doubled the contribution of gluconeogenesis to glucose output in control rats but failed to modify gluconeogenesis in overfed animals (Wang et al. 2001). On the contrary, fasting over several days induced a decrease of $10 \%$ in body weight associated with a $53 \%$ reduction in plasma leptin (Boden 1997). The reduced leptin levels may be a survival response to minimize the energy expenditure during weight loss. The lowered plasma leptin levels also facilitate mobilization of energy stores by promoting secretion of glucocorticoids (Legradi et al. 1997). It is therefore postulated that a drop in plasma leptin levels may be a key short-term adaptation mechanism to starvation or famine situations (Jequier 2002).

Hyperphagia and elevated levels of leptin (along with those of insulin) are common features of obesity (Igel et al. 1997, Lu et al. 1998, Marsh et al. 1999). This seems to be paradoxical since leptin itself is a potent inhibitor of food intake and is expected to decrease insulin levels via improved insulin action and inhibition of insulin secretion (Flier 1997, Rossetti et al. 1997, Sivitz et al. 1997, Shimomura et al. 1999, Wang et al. 1999). It is plausible that leptin may have been somewhat mislabeled as a metabolic hormone that can limit the excessive weight gain of individuals exposed to an environment of plenty. In fact, the hormone may never be able to achieve this goal with the epoch of hyperleptinemia and leptin resistance associated with obesity. Recently, the concept of leptin resistance has been articulated to be 'selective leptin resistance' largely based on the preserved sympathoexcitatory actions of leptin, despite resistance to the satiety and weight-reducing properties of the hormone (Mark et al. 2002). The 'selective leptin resistance' theory is feasible to explain why hyperleptinemia contributes to increased sympathetic activity and arterial pressure in obesity, whereas at the same time there is resistance to the metabolic (satiety and weight-reducing) actions of leptin. If selective leptin resistance occurs in obese humans, then leptin could contribute to the sympathetic overactivity and hypertension despite resistance to its metabolic actions 
(Mark et al. 2002). Although leptin or selective leptin resistance may be accepted as the causative factor for metabolic as well as cardiovascular dysfunctions, the mechanism(s) leading to this resistance has never been elucidated. Using transgenic techniques to overexpress uncoupling protein-1 (UCP-1) in skeletal muscle of agouti yellow obese mice, it was found that the UCP-1 mice displayed increased metabolism, promoted weight loss and increased insulin sensitivity. More importantly, the UCP-1 mice showed decreased arterial pressure, serum leptin and urinary catecholamines. The hypotensive effect of UCP-1 may be blunted by leptin administration (Bernal-Mizrachi et al. 2002). Uncoupling proteins are known to dissipate food metabolism-derived energy as heat rather than being stored as high-energy phosphates. UCP-1 is expressed only in brown adipose tissue where leptin is released. Therefore, it is possible that UCP-1induced regulation of thermogenic metabolism may play a role in the leptin signaling cascade although further study is warranted (Mark \& Sivitz 2002). Last, but not least, leptin resistance can be beneficial in certain situations since it may facilitate energy storage to avoid the consequences of starvation when food resources are limited.

Leptin plays a physiological role but leptin resistance may be pathophysiological for metabolic and cardiovascular dysfunction under obesity. While the role of leptin has been determined in elevation of sympathetic nervous system activity, many of its action on the cardiovascular system such as cardiac growth and ventricular function have not been carefully studied. Understanding the signaling mechanisms behind selective leptin resistance and searching for strategies to alleviate this hormone resistance should have significant clinical value in managing obesity and obesity-associated cardiovascular dysfunction.

\section{Acknowledgements}

The author thanks Dr Bruce Culver for his critical comments on the manuscript. Editorial and secretarial assistance from Karissa $\mathrm{H}$ la Cour, Jennifer An and Anna R Eason is appreciated. The author sincerely apologizes to those whose important work was unable to cite in this review due to space limitation.

\section{Funding}

Work in the author's laboratory is supported by the American Diabetes Association, the American Heart Association, Max Baer Heart Fund and the National Institutes of Health. The author has no conflict of interest regarding this work.

\section{References}

Ahima RS \& Flier JS 2000 Leptin. Annual Review of Physiology 62 413-437.
Air EL, Benoit SC, Clegg DJ, Seeley RJ \& Woods SC 2002 Insulin and leptin combine additively to reduce food intake and body weight in rats. Endocrinology 143 2449-2452.

Aizawa-Abe M, Ogawa Y, Masuzaki H, Ebihara K, Satoh N, Iwai H, Matsuoka N, Hayashi T, Hosoda K, Inoue G, Yoshimasa Y \& Nakao K 2000 Pathophysiological role of leptin in obesity-related hypertension. Journal of Clinical Investigation 105 1243-1252.

Anderson CM \& Ren J 2002 Leptin resistance and endothelial dysfunction in preeclampsia. Cellular and Molecular Biology 48 OL323-OL329.

Atkinson LL, Fischer MA \& Lopaschuk GD 2002 Leptin activates cardiac fatty acid oxidation independent of changes in the AMP-activated protein kinase-acetyl-CoA carboxylase-malonyl-CoA axis. Journal of Biological Chemistry 277 29424-29430.

Barzilai N, Massilon D, Vuguin P, Hawkins M \& Rossetti L 1997 Leptin selectively decreases visceral adiposity and enhances insulin action. Journal of Clinical Investigation 100 3105-3110.

Bernal-Mizrachi C, Weng S, Li B, Nolte LA, Feng C, Coleman T, Holloszy JO \& Semenkovich CF 2002 Respiratory uncoupling lowers blood pressure through a leptin-dependent mechanism in genetically obese mice. Arteriosclerosis, Thrombosis and Vascular Biology 22 961-968.

Bjorbaek C, Uotani S, da Silva B \& Flier JS 1997 Divergent signaling capacities of the long and short isoforms of the leptin receptor. Journal of Biological Chemistry 272 32686-32695.

Bjorbaek C, El-Haschimi K, Frantz JD \& Flier JS 1999 The role of SOCS-3 in leptin signaling and leptin resistance. Journal of Biological Chemistry 274 30059-30065.

Boden G 1997 Role of fatty acids in the pathogenesis of insulin resistance and NIDDM. Diabetes 46 3-10.

Bouloumie A, Marumo T, Lafontan M \& Busse R 1999 Leptin induces oxidative stress in human endothelial cells. FASEB Journal 13 1231-1238.

Campfield L, Smith FJ, Guisez Y, Devos R \& Burn P 1995 Recombinant mouse $\mathrm{OB}$ protein: evidence for a peripheral signal linking adiposity and central neural networks. Science 269 546-549.

Caro JF, Sinha MK, Kolaczynski W, Zhang PL \& Considine RV 1996 Leptin: the tale of an obesity gene. Diabetes 45 1455-1462.

Cases JA, Gabriely I, Ma XH, Yang XM, Michaeli T, Fleischer N, Rossetti L \& Barzilai N 2001 Physiological increase in plasma leptin markedly inhibits insulin secretion in vivo. Diabetes 50 348-352.

Chen ZP, Mitchelhill KI, Michell BJ, Stapleton D, Rodriguez-Crespo I, Witters LA, Power DA, Ortiz de Montellano PR \& Kemp BE 1999 AMP-activated protein kinase phosphorylation of endothelial NO synthase. FEBS Letters 443 285-289.

Correia ML, Haynes WG, Rahmouni K, Morgan DA, Sivitz WI \& Mark AL 2002 The concept of selective leptin resistance: evidence from agouti yellow obese mice. Diabetes 51 439-442.

De Pergola G \& Pannacciulli N 2002 Coagulation and fibrinolysis abnormalities in obesity. Journal of Endocrinological Investigation $\mathbf{2 5}$ 899-904.

Dotsch J, Wagner R, Groschl M, Schoof E, Harig F, Scharf J, Singer H, Katz N, Blum WF, Dorr HG \& Rascher W 2001 Cardiopulmonary bypass surgery does not further increase elevated serum leptin concentrations after major surgery. Pediatric Critical Care Medicine 2 36-39.

Eikelis N, Schlaich M, Aggarwal A, Kaye D \& Esler M 2003 Interactions between leptin and the human sympathetic nervous system. Hypertension 41 1072-1079.

Evans BA, Agar L \& Summers RJ 1999 The role of the sympathetic nervous system in the regulation of leptin synthesis in C57BL/6 mice. FEBS Letters 444 149-154.

Farooqi IS, Keogh JM, Yeo GS, Lank EJ, Cheetham T \& O'Rahilly S 2003 Clinical spectrum of obesity and mutations in the melanocortin 4 receptor gene. New England Journal of Medicine 348 1085-1095. 
Flier JS 1997 Leptin expression and action: new experimental paradigms. PNAS 94 4242-4245.

Frederich RC, Hamann A, Anderson S, Lollmann B, Lowell BB \& Flier JS 1995 Leptin levels reflect body lipid content in mice: evidence for diet-induced resistance to leptin action. Nature Medicine 1 1311-1314.

Friedman JM 2002 The function of leptin in nutrition, weight and physiology. Nutrition Reviews 60 S1-S14.

Friedman JM \& Halaas JL 1998 Leptin and the regulation of body weight in mammals. Nature 395 763-770.

Fruhbeck G 1999 Pivotal role of nitric oxide in the control of blood pressure after leptin administration. Diabetes 48 903-908.

Fujimaki S, Kanda T, Fujita K, Tamura J \& Kobayashi I 2001 The significance of measuring plasma leptin in acute myocardial infarction. Journal of International Medical Research 29 108-113.

Grassi G, Colombo M, Seravalle G, Spaziani D \& Mancia G 1998 Dissociation between muscle and skin sympathetic nerve activity in essential hypertension, obesity and congestive heart failure. Hypertension 31 64-67.

Hall JE, Crook ED, Jones DW, Wofford MR \& Dubbert PM 2002 Mechanisms of obesity-associated cardiovascular and renal disease. American Journal of the Medical Sciences 324 127-137.

Harvey J, McKay NG, Walker KS, Van der Kaay J, Downes CP \& Ashford ML 2000 Essential role of phosphoinositide 3-kinase in leptin-induced K(ATP) channel activation in the rat CRI-Gi insulinoma cell line. Journal of Biological Chemistry 275 4660-4669.

Havel PJ 2000 Role of adipose tissue in body-weight regulation: mechanisms regulating leptin production and energy balance. Proceedings of the Nutrition Society 59 359-371.

Haynes WG 2000 Interaction between leptin and sympathetic nervous system in hypertension. Current Hypertension Reports 2 311-318.

Haynes WG, Morgan DA, Walsh SA, Mark AL \& Sivitz WI 1997 Receptor mediated regional sympathetic nerve activation by leptin. Journal of Clinical Investigation 100 270-278.

Hintz KK, Aberle NS II \& Ren J 2003 Insulin resistance induces hyperleptinemia, cardiac contractile dysfunction but not cardiac leptin resistance in ventricular myocytes. International Journal of Obesity 27 1196-1203.

Igel M, Becker W, Herberg L \& Joost HG 1997 Hyperleptinemia, leptin resistance, and polymorphic leptin receptor in the New Zealand obese mouse. Endocrinology 138 4234-4239.

Illiano G, Naviglio S, Pagano M, Spina A, Chiosi E, Barbieri M \& Paolisso G 2002 Leptin affects adenylate cyclase activity in $\mathrm{H} 9 \mathrm{c} 2$ cardiac cell line: effects of short- and long-term exposure. American Journal of Hypertension 15 638-643.

Jequier E 2002 Leptin signalling. Cellular Signalling 14 655-663.

Julius S, Valentini M \& Palatini P 2000 Overweight and hypertension: a 2-way street? Hypertension 35 807-813.

Kamohara S, Burcelin R, Halaas JL, Friedman JM \& Charron MJ 1997 Acute stimulation of glucose metabolism in mice by leptin treatment. Nature 389 374-377.

Kellerer M, Koch M, Metzinger E, Mushack J, Capp E \& Haring HU 1997 Leptin activated PI-3 kinase in C2C12 myotubes via janus kinase-2 (JAK-2) and insulin receptor substrate-2 (IRS-2) dependent pathways. Diabetologia 40 1358-1362.

Kimura K, Tsuda K, Baba A, Kawabe T, Boh-oka S, Ibata M, Moriwaki C, Hano T \& Nishio I 2000 Involvement of nitric oxide in endothelium-dependent arterial relaxation by leptin. Biochemical and Biophysical Research Communications 273 745-749.

Kolter T, Uphues I \& Eckel J 1997 Molecular analysis of insulin resistance in isolated ventricular cardiomyocytes of obese Zucker rats. American Journal of Physiology. Endocrinology and Metabolism 273 E59-E67.

Konstantinides S, Schafer K, Koschnick S \& Loskutoff DJ 2001 Leptin-dependent platelet aggregation and arterial thrombosis suggests a mechanism for atherothrombotic disease in obesity. Journal of Clinical Investigation 8 1533-1540.
Kuo JJ, Jones OB \& Hall JE 2003 Chronic cardiovascular and renal actions of leptin during hyperinsulinemia. American Journal of Physiology. Regulatory, Integrative and Comparative Physiology 284 R1037-R1042.

Legradi G, Emerson CH, Ahima RS, Flier JS \& Lechan RM 1997 Leptin prevents fasting-induced suppression of prothyrotropin-releasing hormone messenger ribonucleic acid in neurons of the hypothalamic paraventricular nucleus. Endocrinology 138 2569-2576.

Leibel RL, Chung WK \& Chua CS Jr 1997 The molecular genetics of rodent single gene obesities. Journal of Biological Chemistry $\mathbf{2 7 2}$ 31937-31940.

Lembo G, Vecchione C, Fratta L, Marino G, Trimarco V, d'Amati G \& Trimarco B 2000 Leptin induces direct vasodilation through distinct endothelial mechanisms. Diabetes 49 293-297.

Levy JR \& Stevens W 2001 The effects of insulin, glucose and pyruvate on the kinetics of leptin secretion. Endocrinology 142 3558-3562.

von Lewinski D, Voss K, Hulsmann S, Kogler H \& Pieske B 2003 Insulin-like growth factor-1 exerts $\mathrm{Ca}^{2+}$-dependent positive inotropic effects in failing human myocardium. Circulation Research 92 169-176.

Lu H, Duanmu Z, Houck C, Jen KL, Buison A \& Dunbar JC 1998 Obesity due to high fat diet decreases the sympathetic nervous and cardiovascular responses to intracerebroventricular leptin in rats. Brain Research Bulletin 47 331-335.

Malmqvist K, Ohman KP, Lind L, Nystrom F \& Kahan T 2002 Relationships between left ventricular mass and the renin-angiotensin system, catecholamines, insulin and leptin. Journal of Internal Medicine 252 430-439.

Mancuso P, Gottschalk A, Phare SM, Peters-Golden M, Lukacs NW \& Huffnagle GB 2002 Leptin-deficient mice exhibit impaired host defence in Gram-negative pneumonia. Journal of Immunology 168 4018-4024

Margetic S, Gazzola C, Pegg GG \& Hill RA 2002 Leptin: a review of its peripheral actions and interactions. International Journal of Obesity 26 1407-1433.

Mark AL \& Sivitz WI 2002 Uncoupling metabolism and coupling leptin to cardiovascular disease. Arteriosclerosis, Thrombosis and Vascular Biology 22 881-883.

Mark AL, Shaffer RA, Correia MLG, Morgan DA, Sigmund CD \& Haynes WG 1999 Contrasting blood pressure effects of obesity in leptin-deficient $\mathrm{ob} / \mathrm{ob}$ mice and agouti yellow obese mice. Journal of Hypertension 17 1949-1953.

Mark AL, Correia ML, Rahmouni K \& Haynes WG 2002 Selective leptin resistance: a new concept in leptin physiology with cardiovascular implications. Journal of Hypertension 20 1245-1250.

Marsh DJ, Hollopeter G, Huszar D, Laufer R, Yagaloff KA, Fisher SL, Burn P \& Palmiter RD 1999 Response of melanocortin-4 receptor-deficient mice to anorectic and orexigenic peptides. Nature Genetics 21 119-122.

Matsumura K, Abe I, Tsuchihashi T \& Fujishima M 2000 Central effects of leptin on cardiovascular and neurohormonal responses in conscious rabbits. American Journal of Physiology. Regulatory, Integrative and Comparative Physiology 278 R1314-R1320.

Minokoshi Y, Kim YB, Peroni OD, Fryer LG, Muller C, Carling D \& Kahn BB 2002 Leptin stimulates fatty-acid oxidation by activating AMP-activated protein kinase. Nature 415 339-343.

Mitchell JL, Morgan DA, Correia ML, Mark AL, Sivitz WI \& Haynes WG 2001 Does leptin stimulate nitric oxide to oppose the effects of sympathetic activation? Hypertension 38 1081-1086.

Modan-Moses D, Ehrlich S, Kanety H, Dagan O, Pariente C, Esrahi N, Lotan D, Vishne T, Barzilay Z \& Paret G 2001 Circulating leptin and the perioperative neuroendocrinological stress response after pediatric cardiac surgery. Critical Care Medicine 29 2377-2382.

Mueller WM, Gregoire FM, Stanhope KL, Mobbs CV, Mizuno TM, Warden CH, Stem JS \& Havel PJ 1998 Evidence that glucose metabolism regulates leptin secretion from cultured rat adipocytes. Endocrinology 139 551-558. 
Naderali EK, Brown MJ, Pickavance LC, Wilding JP, Doyle PJ \& Williams G 2001 Dietary obesity in the rat induces endothelial dysfunction without causing insulin resistance: a possible role for triacylglycerols. Clinical Science 101 499-506.

Nakagawa K, Higashi Y, Sasaki S, Oshima T, Matsuura H \& Chayama K 2002 Leptin causes vasodilation in humans. Hypertension Research 25 161-165.

Narkiewicz K, Somers VK, Mos L, Kato M, Accurso V \& Palatini P 1999 An independent relationship between plasma leptin and heart rate in untreated patients with essential hypertension. Journal of Hypertension 17 245-249.

Nickola MW, Wold LE, Colligan PB, Wang GJ, Samson WK \& Ren J 2000 Leptin attenuates cardiac contraction in rat ventricular myocytes. Role of NO. Hypertension 36 501-505.

Northcott CA, Poy MN, Najjar SM \& Watts SW 2002 Phosphoinositide 3-kinase mediates enhanced spontaneous and agonist-induced contraction in aorta of deoxycorticosterone acetate-salt hypertensive rats. Circulation Research 91 360-369.

Ookuma M, Ookuma K \& York D 1998 Effects of leptin on insulin secretion from isolated rat pancreatic islets. Diabetes 47 219-223.

Parhami F, Tintut Y, Ballard A, Fogelman AM \& Demer LL 2001 Leptin enhances the calcification of vascular cells: artery wall as a target of leptin. Circulation Research 88 954-960.

Pelleymounter MA, Cullen MJ, Baker MB, Hecht R, Winters D, Boone T \& Collins F 1995 Effects of the obese gene product on body weight regulation in ob/ob mice. Science $269540-543$.

Quehenberger P, Exner M, Sunder-Plassmann R, Ruzicka K, Bieglmayer C, Endler G, Muellner C, Speiser W \& Wagner O 2002 Leptin induces endothelin-1 in endothelial cells in vitro. Circulation Research 90 711-718.

Ren J, Sowers JR, Walsh MF \& Brown RA 2000 Reduced contractile response to insulin and IGF-I in ventricular myocytes from genetically obese Zucker rats. American Journal of Physiology. Heart and Circulatory Physiology 279 H1708-H1714.

Rossetti L, Massillon D, Barzilai N, Vuguin P, Wu J, Liu R \& Wang J 1997 Short-term effects of leptin on hepatic gluconeogenesis and in vivo insulin action. Journal of Biological Chemistry 272 27758-27763.

Satoh N, Ogawa Y, Katsuura G, Numata Y, Tsuji T, Hayase M, Ebihara K, Masuzaki H, Hosoda K, Yoshimasa Y \& Nakao K 1999 Sympathetic activation of leptin via the ventromedial hypothalamus: leptin-induced increase in catecholamine secretion. Diabetes $\mathbf{4 8}$ 1787-1793.

Schulze PC, Kratzsch J, Linke A, Schoene N, Adams V, Gielen S, Erbs S, Moebius-Winkler S \& Schuler G 2003 Elevated serum levels of leptin and soluble leptin receptor in patients with advanced chronic heart failure. European Journal of Heart Failure 5 33-40.

Shek EW, Brands MW \& Hall JE 1998 Chronic leptin infusion increases arterial pressure. Hypertension 31 409-414.

Shimomura I, Hammer RE, Ikemoto S, Brown MS \& Goldstein JL 1999 Leptin reverses insulin resistance and diabetes mellitus in mice with congenital lipodystrophy. Nature 401 73-76.

Shirasaka T, Takasaki M, \& Kannan H 2003 Cardiovascular effects of leptin and orexins. American Journal of Physiology. Regulatory, Integrative and Comparative Physiology 284 R639-R651.

Sierra-Honigmann MR, Nath AK, Murakami C, Garcia-Cardena G, Papapetropoulos A, Sessa WC, Madge LA, Schechner JS, Schwabb MB, Polverini PJ \& Flores-Riveros JR 1998 Biological action of leptin as an angiogenic factor. Science 281 1683-1686.

Singhal A, Farooqi IS, Cole TJ, O'Rahilly S, Fewtrell M, Kattenhorn M, Lucas A \& Deanfield J 2002 Influence of leptin on arterial distensibility: a novel link between obesity and cardiovascular disease? Circulation 106 1919-1924.
Sivitz WI, Walsh SA, Morgan DA, Thomas MJ \& Haynes WG 1997 Effects of leptin on insulin sensititvity in normal rats. Endocrinology 138 3395-3401.

Sowers JR 1998 Obesity and cardiovascular disease. Clinical Chemistry 44 1821-1825.

Steinberg HO, Chaker H, Leaming R, Johnson A, Brechtel G \& Baron AD 1996 Obesity/insulin resistance is associated with endothelial dysfunction: implications for the syndrome of insulin resistance. Journal of Clinical Investigation 97 2601-2610.

Steinberg GR, Rush JW \& Dyck DJ 2003 AMPK expression and phosphorylation are increased in rodent muscle after chronic leptin treatment. American Journal of Physiology. Endocrinology and Metabolism 284 E648-E654.

Sweeney G 2002 Leptin signalling. Cellular Signalling 14 655-663.

Trayhurn P, Duncan JS, Hoggard N \& Rayner DV 1998 Regulation of leptin production: a dominant role for the sympathetic nervous system? Proceedings of the Nutrition Society 57 413-419.

Unger RH \& Orci L 2002 Lipoapoptosis: its mechanism and its diseases. Biochimica et Biophysica Acta 1585 202-212.

Vecchione C, Maffei A, Colella S, Aretini A, Poulet R, Frati G, Gentile MT, Fratta L, Trimarco V, Trimarco B \& Lembo G 2002 Leptin effect on endothelial nitric oxide is mediated through Akt-endothelial nitric oxide synthase phosphorylation pathway. Diabetes 51 168-173.

Wang J, Liu R, Liu L, Chowdhury R, Barzilai N, Tan J \& Rossetti L 1999 The effect of leptin on Lep expression is tissue-specific and nutritionally regulated. Nature Medicine 5 895-899.

Wang J, Obici S, Morgan K, Barzilai N, Feng Z \& Rossetti L 2001 Overfeeding rapidly induces leptin and insulin resistance. Diabetes 50 2786-2791.

Winnicki M, Phillips BG, Accurso V, van De Borne P, Shamsuzzaman A, Patil K, Narkiewicz K \& Somers VK 2001 Independent association between plasma leptin levels and heart rate in heart transplant recipients. Circulation 104 384-386.

Winnicki M, Somers VK, Accurso V, Phillips BG, Puato M, Palatini P \& Pauletto P 2002 Fish-rich diet, leptin, and body mass. Circulation 106 289-291.

Wold, LE, Relling DP, Duan J, Norby FL \& Ren J 2002 Abrogated leptin-induced cardiac contractile response in ventricular myocytes under spontaneous hypertension: role of Jak/STAT pathway. Hypertension 39 69-74.

Yamagishi S, Edelstein D, Du X, Kaneda Y, Guzman M \& Brownlee M 2001 Leptin induces mitochondrial superoxide production and monocyte chemoattractant protein-1 expression in aortic endothelial cells by increasing fatty acid oxidation via protein kinase A. Journal of Biological Chemistry 276 25096-25100.

Zhang Y, Proenca R, Maffei M, Barone M, Leopold L \& Friedman J 1994 Positional cloning of the mouse obese gene and its human homologue. Nature 372 425-432.

Zhang W, Telemaque-Potts S, Anderson PR, Wang Z, An J, Newgard CB \& Victor RG 2002 Adenoviral leptin as gene therapy for obesity related hypertension. American Journal of Hypertension 15 $1 \mathrm{~A}$ (Abstract).

Zhao AZ, Bornfeldt KE \& Beavo JA 1998 Leptin inhibits insulin secretion by activation of phosphodiesterase 3B. Journal of Clinical Investigation 102 869-873.

Received in final form 21 August 2003

Accepted 28 October 2003 